\title{
Neural Network and GA based Intelligent B2B Negotiation System
}

\author{
Alexander T \\ Research Scholar \\ Dept. of Computer Science \\ Bharathidasan University \\ Tiruchirappalli, India.
}

\author{
E. Kirubakaran, PhD. \\ Additional General Manager \\ SSTP (Systems) \\ Bharat Heavy Electricals Ltd \\ Tiruchirappalli, India.
}

\begin{abstract}
E-commerce has become one of the most important aspects of today's buying and selling process. Due to the large number of transactions performed online, there arises a need for intelligent support tools to improve the efficiency in performing these transactions. According to the BBT business model, negotiation plays an important part in B2B ecommerce. Web services play a major role in performing the negotiations. Here a two phase system is proposed that helps in appropriate web service discovery and service analysis and finally provides the user with a set of workflows that are appropriate for the current user. A neural network based analyzer is used in the discovery phase, which helps in selecting the appropriate web service. Finally, the GA based analyzer is used for resolving the final $\mathrm{k}$ best workflows.
\end{abstract}

\section{Keywords}

Automated negotiations, E-commerce, web services, neural networks.

\section{INTRODUCTION}

The advent of e-business has changed the entire business process and in turn, the traditional negotiation process. In accordance with e-business, negotiation refers to the process of getting into an electronic agreement, that is acceptable by both the seller and the buyer [1]. This agreement is an automated process of the traditional negotiation process. Due to the presence of automation, users have the possibility to negotiate with multiple sellers or buyers at the same time, hence reducing the amount of time and resources spent. But, the process of automating this negotiation process is not simple. It provides the system with a complex space with a strict deadline criteria. Further, multiple constraints are to be met and information provided might not always be perfect [2]. Hence, the automated system that is to be designed should adapt to these criteria and meet the goals of the user. The system should also be flexible enough to be integrated to data mining systems for information researching and information retrieval[3]. It should also be able to carry out goal driven multi negotiations with multiple issues at the same time[4], create user profiles[5] ,support vital negotiation mechanism[6] and create a hybrid negotiation system[3].

\subsection{B2B ECOMMERCE}

Business-to-business (B2B) refers to commerce transactions that take place between businesses. These can be transactions that occur between a manufacturer and a dealer, or between a dealer and a retailer. In general, there are many B2B
(Business-to-Business) transactions taking place [7][8][9]. The primary reason for this is that in a supply chain there will be many business transactions, that require raw materials or partially completed components, while the final product is provided to just one customer and the chain ends there. For example, an automobile manufacturer makes several B2B transactions such as buying tires, glass for windscreens, and rubber hoses for its vehicles.

\subsection{Stages of Ecommerce}

According to the BBT model [10], B2B business process can be divided into six important stages. The first stage is "Partnership Formation" which involves finding the business partners that provide products or services in a supply chain as well as the forming of a virtual enterprise; the second stage is "Brokering" which is the process of matching sellers who supply goods or services to the buyers who require them; the third stage is "Negotiation" where the traders aim to reach an agreement about what actions should be performed under what conditions; the fourth stage "Contract Formation" marks the end of negotiation and involves the agreed terms being put into a legally binding contract; the fifth stage "Contract Fulfilment" refers to the parties executing the agreed transactions according to the terms specified in the contract; "Service Evaluation" is the final stage where traders evaluate their satisfaction with the transactions so as to prepare for another partnership formation in the future. The proposed WSDNS system architecture directly supports "'Partnership Formation", "Brokering", "Negotiation" and "Contract Formation". Moreover, it can be easily extended to support the last two stages of the BBT model.

\subsection{Neural Networks}

An artificial neural network (ANN), [11]\&[12] often just called a "neural network" (NN), is a mathematical model or computational model based on biological neural networks, i.e. it functions similar to that of a biological neural system. It consists of an interconnected group of nodes called artificial neurons and these nodes processes information and provide the output to the user. These nodes communicate with each other and every node has an assigned functionality and it performs the process on the data provided to it. 


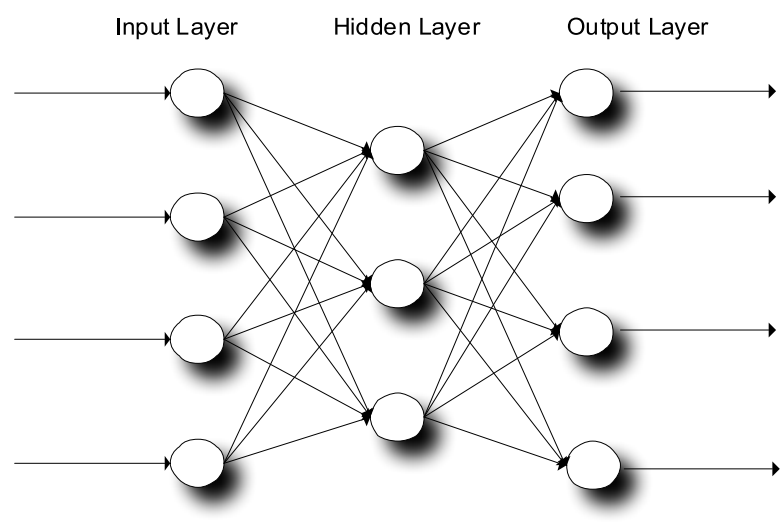

Fig 1: Structure of an Artificial Neural Network

The ANN changes its structure based on external or internal information that flows through the network during the learning phase. A basic ANN consists of three layers, the input, hidden and the output layers. The hidden layer performs the processing in the network.

\subsection{Genetic Algorithms}

Genetic algorithm (GA) is a search heuristic that mimics the process of natural evolution. This heuristic is routinely used to generate useful solutions to optimization and search problems.[13] Genetic algorithms belong to the larger class of evolutionary algorithms (EA), which generate solutions to optimization problems using techniques inspired by natural evolution, such as inheritance, mutation, selection, and crossover.

Selection is the stage of a genetic algorithm in which individual genomes are chosen from a population for later breeding (recombination or crossover). A generic selection evaluates the fitness function for each individual, providing fitness values, which are then normalized. Normalization means dividing the fitness value of each individual by the sum of all fitness values, so that the sum of all resulting fitness values equals 1 . The population is sorted by descending fitness values. A random number $\mathrm{R}$ between 0 and 1 is chosen. The selected individual is the first one whose accumulated normalized value is greater than $R$.

Stochastic universal sampling (SUS) [20] is a technique used in genetic algorithms for selecting potentially useful solutions for recombination. SUS is a development of fitness proportionate selection (FPS) which exhibits no bias and minimal spread. SUS uses a single random value to sample all of the solutions by choosing them at evenly spaced intervals. This gives weaker members of the population (according to their fitness) a chance to be chosen and thus reduces the unfair nature of fitness-proportional selection methods.

The remaining paper is structured as follows. Section 3 provides related works regarding the e-commerce systems and Section 4 provides the overall system architecture. Section 5 describes the complete negotiation mechanism along with its sub structures.

\section{RELATED WORKS}

Existing e-negotiation process employs intelligent agents that analyzes and processes based on the price [1]. While in real world, price alone cannot be taken as a constraint. Constraints for negotiations involve multiple items such as price, quantity, quality, availability, etc. [14]. In [15] a fuzzy similarity based trade-off mechanism is proposed to search for near optimal negotiation solutions based on realistic assumptions. The solution based on a bilateral negotiation, whereas negotiation in e-commerce often involves multiple parties.

Genetic algorithm has also been applied to learn effective rules to support the negotiation processes [16]. In this process, each chromosome is presented a rule. Its fitness is measured by the number of time the particular chromosome has been used during the decision making process. To present this in an efficient manner, each negotiator's preference is hypothesized or if the value is available, it is used. Hence the user needs to know the complete knowledge of the search space before beginning the process. The returned results have a higher probability to be biased depending upon the hypothesis being used.

\section{SYSTEM ARCHITECTURE}

It is a necessity for the $\mathrm{B} 2 \mathrm{~B}$ e-commerce systems to be efficient and accurate, due to the amount of money involved. Therefore, negotiation agents should be developed based on the assumption of bounded rather than perfect rationality [17]. Since businesses have the necessity to include their own preferences, such as negotiation strategies with respect to their business partners, the developed negotiation mechanism should have a distributed and a flexible structure. As the user only knows his own preferences but not the preferences of its opponents [18][19] in typical B2B negotiation settings, negotiation agents must be able to make sensible decisions based on incomplete and uncertain information. Since B2B negotiations often involve multiple parties who will exploit many issues (e.g., price, quantity, product quality, etc.) simultaneously, practical negotiation mechanisms must be able to support multi-lateral multi-issue negotiations. 


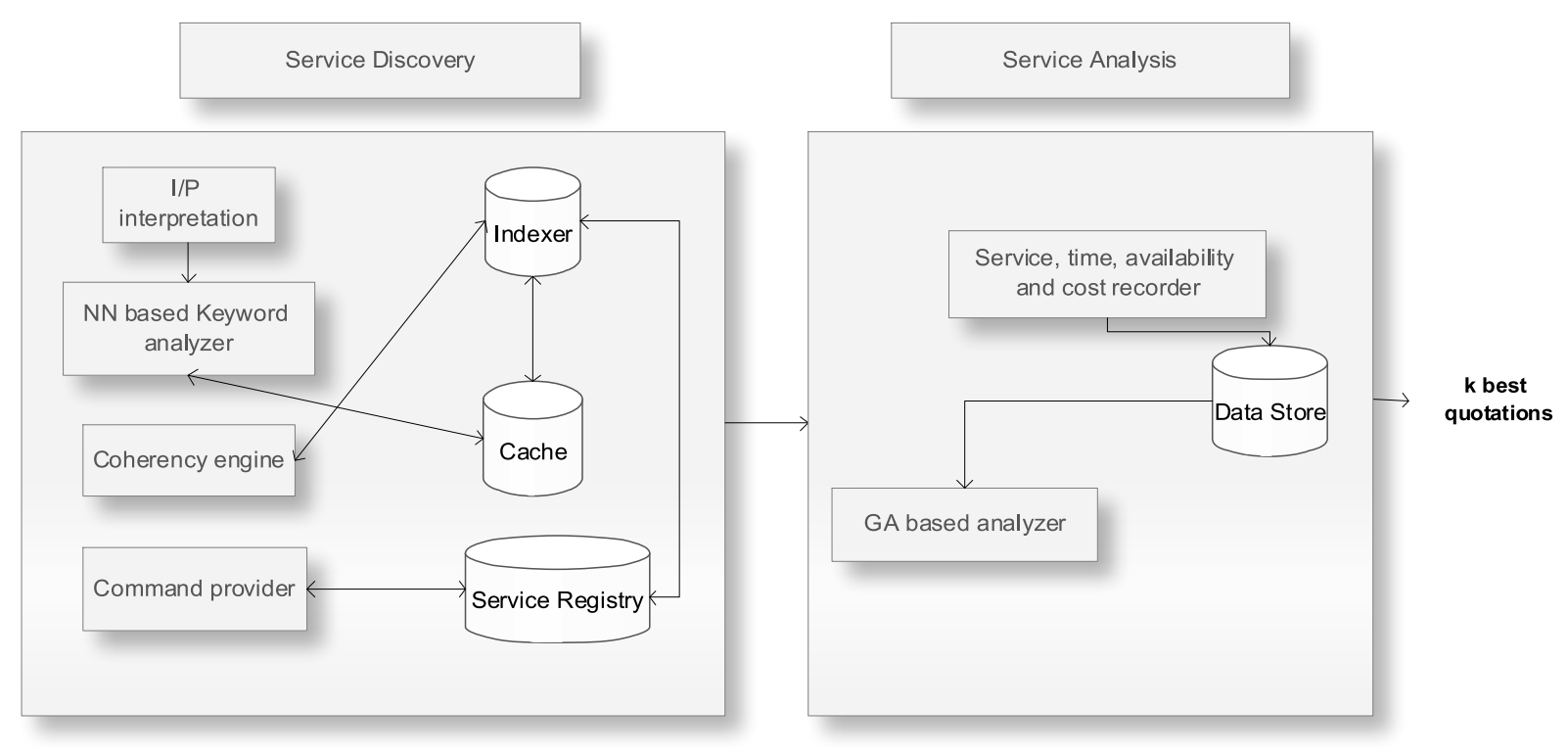

\section{Fig 2: System architecture}

Fig shows the complete architecture of our system. Our proposed system initially performs service discovery, which searches the available services and shortlists all the appropriate services. All the sub process that are involved under the base process are initially listed. Every sub process has the probability of being performed by may web services. Hence, a keyword match is performed for the selection of appropriate web services, after which storage of current details is performed for future use. The details about the web service and the provider are then passed on to the next phase. The next phase performs analysis of these services based on the appropriate properties (cost, time, availability, quantity and quality). Selection of the appropriate services is performed by Stochastic Universal Sampling (SUS) method. This method selects the appropriate candidates, after which the workflow is designed.

B2B processes are never atomic. Every process intrinsically contains many sub-processes. Completion of every subprocess is performed by a web service. The workflow is created by organizing these web services in an orderly manner. Every atomic process is performed by many web services, hence the user can create many such workflows. All these workflows are analyzed to provide the user with $\mathrm{k}$ best quotations, where the value for $\mathrm{k}$ is defined by the user.

\section{NEGOTIATION MECHANISM}

The negotiation mechanism is performed in two stages. The first stage performs the discovery of appropriate services and the second phase performs the analysis of these shortlisted services to provide the user with the requested number of workflows.

\subsection{Service Discovery}

The service discovery phase receives the input from the user, breaks down the input into atomic processes and finds the appropriate web services that perform the tasks. It also performs the search optimization process that helps in providing quicker response to the query presented to the system.

\subsubsection{Input Interpretation}

The input that is provided to the system is analyzed and is converted to a format that is understood by the neural networks. The converted input is provided to the neural networks for further analysis.

\subsubsection{Neural Network Based Keyword Analyzer}

The input provided is in the form of distinct entities. The input phase takes in these entities and passes them to the processing phase. These data are analyzed in the processing phase. This phase initially divides the keywords and checks if the provided input contains commonly occurring words. These words are eliminated for optimizing the search process, and the data is passed on to the next phase for processing. The remaining words are considered to be the words of importance. Redundancies are checked, and in case of redundancies, they are eliminated. This process helps in faster processing. These words are processed and the corresponding web services are discovered.

\subsubsection{Indexer}

The indexer helps in organization of available information for faster retrieval. All information interpreted by the neural network system is stored in the system along with the details about the web services and is provided an index value. If the same data is again encountered by the $\mathrm{NN}$, then it can be directly retrieved from the storage.

\subsubsection{Cache}

All the recently accessed web services along with their keywords are stored in the cache. The cache contains categories and all the recently accessed information in the particular category are stored in the cache. This makes it easier and faster for the users to avail the required information without going through the entire process. 


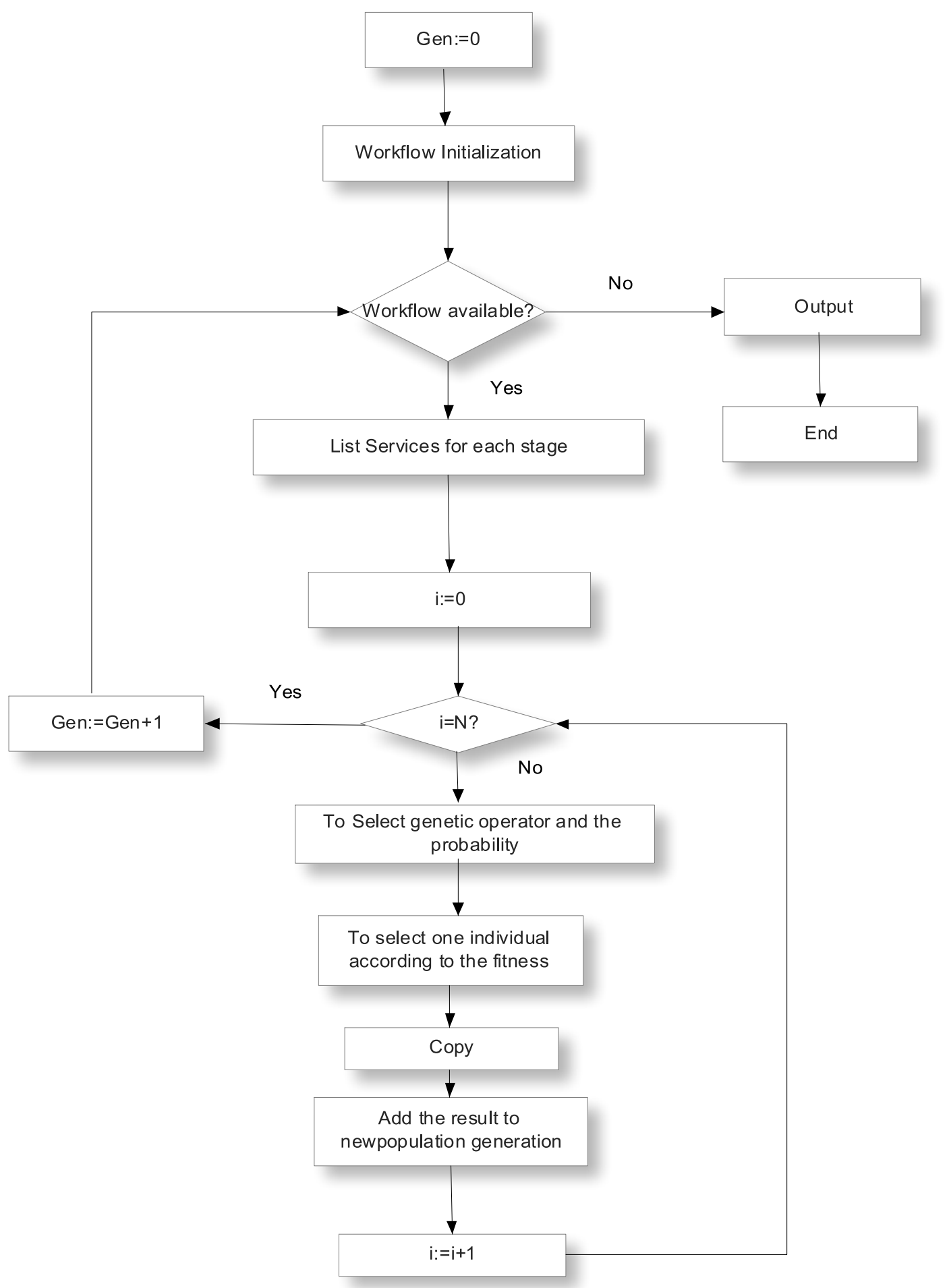

Figure 3: GA Based Analyzer

\subsubsection{Coherency Engine}

Some services may be withdrawn by the publishers, or updation of services might take place. These might lead to inconsistency in the stored data. The coherency engine keeps track of the availability of all the indexed services and keeps them up to date. This process is performed regularly to maintain consistency in the system.

\subsubsection{Service Registry}

Registry contains information about all the hoisted web services and their properties. Many service providers provide the same service but at different cost and time. All these details are recorded in the service registry for future selection of the appropriate web services. 


\subsubsection{Command Provider}

The command provider helps in publishing the web service to the service registry. Through the command provider, the web service providers can provide save, update and delete commands to their corresponding web services.

\subsection{Service Analysis}

All the appropriate services that have been discovered in the previous phase are passed on to the service analysis phase. This phase creates a workflow based on the user's requirements and the available services.

\subsubsection{Attribute Recorder}

All the shortlisted web services are subjected to an initial analysis and property check. Services that satisfy the minimum requirements of the user on the basis of time, availability, quality, quantity and cost are filtered. The complete workflow is built, after which all services corresponding to each phase of the workflow are listed and transferred to the GA based analyzer for workflow construction.

\subsubsection{GA Based Analyzer}

The GA based analyzer uses Stochastic Universal Sampling (SUS)[20] for selection of potentially useful solutions for the workflow building. Initially, the complete sequence or the workflow is defined, and then the fitness value of all the shortlisted services is evaluated. Every stage of the workflow is selected and its corresponding services are listed. A service is selected at random from the current service pool and its fitness value is evaluated to check if it has the required fitness (if it satisfies the required constraints). If so the service is included for building the workflow. Similarly, all the stages in the workflow are evaluated and corresponding services are assigned. This process is carried out for all the requested number of workflows. Negotiation is performed in each of these workflows and finally, the fitness value of all the workflows is evaluated and $\mathrm{k}$ best workflows are chosen for providing to the user.

A negotiation space Neg $=\{\mathrm{P}, \mathrm{A}, \mathrm{D}, \mathrm{U}, \mathrm{T}\}$ is a 5-tuple which consists of a finite set of negotiation parties (agents) $\mathrm{P}$, a set of attributes A understood by all the parties $p \in P$, a set of attribute domains $\mathrm{D}$ for $\mathrm{a} \epsilon \mathrm{A}$, and a set of utility functions $\mathrm{U}$ with each function for an agent $\mathrm{p} \in \mathrm{P}$. An attribute domain is denoted $D_{a i}$ where $D_{a i} \in D$ and $a_{i} \in A$. An utility function pertaining to an agent $\mathrm{p}$ is defined by: $\mathrm{U}_{\mathrm{p}}^{\mathrm{o}}: \mathrm{D}_{\mathrm{a} 1} \times \mathrm{D}_{\mathrm{a} 2} \times \ldots$ $\mathrm{D}_{\text {an }} \Rightarrow[0,1]$. Each agent $\mathrm{p}$ has a deadline $\mathrm{t}_{\mathrm{p}}^{\mathrm{p}} \in \mathrm{T}$. It is assumed that information about $\mathrm{P}, \mathrm{A}, \mathrm{D}$ is exchanged among the negotiation parties during the ontology sharing stage before negotiation actually takes place.

Each offered service contains a set of offers $o=\left\{d_{1}, d_{2}, \ldots . . d_{n}\right\}$. These represent the offers that are available for the service requestor. After initial discovery, the offer that is considered optimal by the service provider is presented to the requestor. This marks the beginning of the negotiation process. The requestor then presents a counter offer. The transfer of offers and counter offers are performed till the receiver quits or accepts the offer.

As the maximum number of workflows that can be created using a set of web services is very high, we provide the termination criteria hardcoded. This helps in stopping at a particular instant, rather than performing infinite iterations.

\section{Algorithm:}

Input: Base process

Output: k best workflows

Analyze input process and divide it into appropriate format Pass the data to the input phase of the neural networks

For each input

Check if the word is a common phrase

If yes, skip to next word

Else,

Translate input

Using the Indexer, check if the requested service is available in the cache

If yes, return the Service

Else

Check the Service Registry for the Service

If found,

Save the details in the Cache

Add an Indexer entry

Return the Service

List all the properties of the shortlisted Services

Define the workflow stages

Perform the following until $\mathrm{k}$ different workflows

For every workflow stage

List the services that perform the process

Select the optimal service using SUS

Perform negotiation and if passed, proceed with next workflow.

\section{CONCLUSIONS}

Negotiation is one of the most important process that is carried out during any B2B transaction. These help in the advancement of e-commerce and pave way for the next generation intelligent business systems. Since B2B negotiations are characterized by combinatorial complex negotiation spaces, tough negotiation deadlines and limited information about the opponents, practical negotiation mechanisms must be able to address these issues. The proposed Web services and intelligent agents-based negotiation system fulfils most of the requirements of practical negotiation systems.

The existing B2B analysis systems provide the users with an analyzer that presents the best available results to the user, while the genetic based analyzer provides the user with new optimal sequences that have the least opportunity of being mined by non genetic algorithms. Simulations results show a considerable performance improvement when dealing with the negotiation. Better and faster negotiations were observed which provides close resemblance to the real time scenarios. 
Further, usage of neural networks in the initial phase reduced the amount of time consumed for identifying the process. Usage of neural networks and genetic based methodologies provides us with optimal results, which outperform their counterparts. Our experiments show that the agents developed in our system provides optimal and fast results. Future work includes the improvement of the adaptation level of our intelligent agents so that they can take into account the dynamic negotiation behavior of their negotiation opponents during bargaining.

\section{REFERENCES}

[1] Peter Braun, Jakub Brzostowski1, Gregory Kersten, Jin Baek Kim, Ryszard Kowalczyk1, Stefan Strecker, and RustamVahidov, 2006, "Intelligent Decision-Making Support Systems: Foundations, Applications And Challenges", Chapter 15, pp. 271-300, Springer, London.

[2] Philippe Pasquier, Ramon Hollands, IyadRahwan, 2010, "An Empirical Study Of Interest-Based Negotiation", Proceeding of ninth international conference on electronic commerce" Published online Springer.

[3] Ying Lei; Shijun Zhang, "Effective Automated Negotiation in Electronic Marketplace", IEEE. 2008.

[4] Thalerngsak.Kijthaweesinpoon and Pei Zhou, 2008, "An Extended Unified Negotiation Model for Ecommerce Systems", International Symposium on Information Science and Engineering, IEEE, pp.758-761.

[5] Thalerngsak.Kijthaweesinpoon and Pei.Zhou, 2008, "An Automated Mechanism For Negotiations in Ecommerce Systems", International Seminar on Business and Information Management, pp.194-198,IEEE.

[6] Raymond Lau, "Towards A Web Services And Intelligent Agents-Based Negotiation System for B2b eCommerce", 2007, Electronic Commerce Research and Applications 6, pp.260-273, IEEE.

[7] Sandhusen, Richard, "Marketing". Hauppauge, 2008, "N.Y: Barron's Educational Series". p. 520. ISBN 07641-3932-0.

[8] Shelly, Gary, "Systems Analysis and Design", 2011, Boston, "MA: Course Technology", Cengage Learning. p. 10. ISBN 0-538-47443-2.

[9] Garbade, Michael, Differences in Venture Capital Financing of U.S., UK, 2011, German and French Information Technology Start-ups A Comparative Empirical Research of the Investment Process on the Venture Capital Firm Level. München: GRIN Verlag GmbH. p. 31. ISBN 3-640-89316-6.
[10] M. He, N.R. Jennings, H. Leung, 2003, On AgentMediated Electronic Commerce, IEEE Transactions on Knowledge and Data Engineering, 15 (4), 985-1003, IEEE.

[11] Ms.Smita.Nirkhi, "Potential Use of Artificial Neural Network In Data Mining”, 978-1-4244-5586-7/10, 2010 IEEE.

[12] Zhijun Chen, "Research of Data Mining Based On Neural Network", 978-1-4244-7161-4/10, 2010 IEEE

[13] Atanassov K., H. Aladjov, 2000, "Generalized Nets in Artificial Intelligence", Vol. 2: "Generalized Nets and Machine Learning", Prof. M. Drinov Academic Publishing House, Sofia.

[14] Li Liu, Yan Ma, Cuimei Wen, 2008, "Multilateral MultiIssue Automated Negotiation Model Based on GA", International Conference on Intelligent Computation Technology and Automation, pp.85-89.

[15] Deng-Neng Chen, 2008, "An Agent-Based Model For Consumer-To-Business Electronic Commerce", An International Journals Expert Systems with Application, 34, Elsevier Ltd, pp.469-481.

[16] S. Matwin, T. Szapiro, K. Haigh, 1991, "Genetic Algorithm Approach to A Negotiation Support System", IEEE Transactions on Systems Man and Cybernetics, 21 (1), 102-114

[17] A.R. Lomuscio, N.R. Jennings, A classification scheme for negotiation in electronic commerce, Journal of Group Decision and Negotiation, 12 (1), 31-56, 2003.

[18] N.R. Jennings, P. Faratin, A.R. Lomuscio, S. Parsons, C. Sierra, M. Wooldridge, 2001, "Automated Negotiation: Prospects, Methods And Challenges", Journal of Group Decision and Negotiation, 10 (2), 199- 215.

[19] P. Faratin, C. Sierra, N.R. Jennings, 2002, "Using Similarity Criteria To Make Issue Trade-Offs In Automated Negotiations", Artificial Intelligence, 142 (2), 205-237.

[20] Tania Pencheva, KrassimirAtanassov, Anthony Shannon, 2009, "Modelling of a Stochastic Universal Sampling Selection Operator in Genetic Algorithms Using Generalized Nets", Tenth Int. Workshop on Generalized Nets Sofia, 1-7. 